\title{
Applications of fluorescence lifetime imaging in clinical medicine
}

\author{
Zhanwen Wang*, Yanping Zheng*, Deqiang Zhao ${ }^{\dagger}$, Ziwei Zhao*, \\ Lixin Liu ${ }^{\S}$, Artem Pliss" , Feiqi Zhull, Jun Liu**, Junle $\mathrm{Qu}^{+, \dagger \dagger}$ \\ and Ping Luan*,\|, \\ ${ }^{*}$ Shenzhen University Health Science Center \\ Shenzhen 518060, P. R. China \\ ${ }^{\dagger}$ Department of Neurology \\ Nanfang Hospital of Southern Medical University \\ Guangzhou, Guangdong 510515, P. R. China \\ $\star$ Key Laboratory of Optoelectronic Devices and \\ Systems of Ministry of Education and Guangdong Province \\ Shenzhen University, Shenzhen 518060, P. R. China \\ ${ }^{\S}$ School of Physics and Optoelectronic Engineering \\ Xidian University, Xi'an 710071, P. R. China \\ "Institutue for Laser, Photonics and Biophotonics \\ State University of New York, Buffalo, New York, USA \\ "Cognitive Impairment Ward of Neurology Department \\ The 3rd Affiliated Hospital of Shenzhen University \\ Shenzhen 518001, P. R. China \\ **Department of Neurodegenerative Diseases and Aging \\ Sun Yat-Sen Memorial Hospital \\ Sun Yat-Sen University, Guangdong, P. R. China \\ †qujunle@szu.edu.cn \\ カluanping@szu.edu.cn
}

Received 8 June 2017

Accepted 21 July 2017

Published 22 August 2017

Fluorescence lifetime is not only associated with the molecular structure of fluorophores, but also strongly depends on the environment around them, which allows fluorescence lifetime imaging microscopy (FLIM) to be used as a tool for precise measurement of the cell or tissue

\$Corresponding author.

Z. Wang and Y. Zheng contributed equally to this work and are considered as co-first authors.

This is an Open Access article published by World Scientific Publishing Company. It is distributed under the terms of the Creative Commons Attribution 4.0 (CC-BY) License. Further distribution of this work is permitted, provided the original work is properly cited. 
microenvironment. This review introduces the basic principle of fluorescence lifetime imaging technology and its application in clinical medicine, including research and diagnosis of diseases in skin, brain, eyes, mouth, bone, blood vessels and cavity organs, and drug evaluation. As a noninvasive, nontoxic and nonionizing radiation technique, FLIM demonstrates excellent performance with high sensitivity and specificity, which allows to determine precise position of the lesion and, thus, has good potential for application in biomedical research and clinical diagnosis.

Keywords: Fluorescence lifetime; fluorescence lifetime imaging microscopy; clinical medicine.

\section{Introduction}

The components of the microenvironment during cell survival include the intercellular substance of the organism and its body fluid. An appropriate microenvironment is the most important condition for normal proliferation, differentiation, metabolism and other functional activities of cells, and without considering this microenvironment, intracellular and intercellular information transfer cannot be assessed. ${ }^{1}$ Thus, biomedical research on the microenvironmental changes in cells or tissues is of great significance. $^{2}$

Methods used in biomedical research require high spatial resolution to identify damaged and normal tissues, high sensitivity to monitor changes in the microenvironment, and high precision to accurately determine those microenvironmental changes. $^{3-5}$ The combination of fluorescence labeling and optical imaging is currently the most commonly used method in biological research. However, it is easily affected by factors such as the intensity of the excited emission, the quenching and uneven distribution of fluorophores; it is difficult to quantify changes in the microenvironment with the fluorescence intensity measured in arbitrary units. The fluorescence lifetime is not affected by the above factors, and it can reflect and quantify the changes in microenvironment of the fluorophore, being measured in absolute units. These features allow for fluorescence lifetime imaging microscopy (FLIM) to accurately quantitatively assess changes of many biophysical parameters of a target molecular microenvironment, such as oxygen pressure, polarity, $\mathrm{pH}$, ion concentration and other biochemical parameters. $^{6,7}$

At present, the gold standard for the diagnosis of organ tissue tumors is pathological diagnosis and for cardiovascular disease, especially coronary artery disease, is angiography. However, many diagnoses of nonneoplastic diseases do not have the so-called gold standard, such as psoriasis, retinal artery occlusion, liver disease, nervous system, digestive system diseases, Alzheimer's disease, etc. Their diagnostic means mostly are clinical symptoms observation, physical examination, and related auxiliary examination, such as ophthalmoscopy, ultrasound, CT, nuclear magnetic resonance, blood examination, endoscopy, scale evaluation and other means of diagnosis, that are subjective and lack specificity. Pathological examination, requires the extraction of living tissue and histological staining, followed by microscopic examination. The extracted tissues and staining affect the worker's judgment and the standard of judgment is also related to the professional level of observation. Angiography has an allergy risk, and the patient is exposed to radiation. FLIM has the advantages of real-time, fast, accurate, nontoxic and so on. On the other hand, intravascular ultrasound is known to be a minimally invasive diagnostic method that does not involve ionizing radiation. Combined with FLIM, it can better observe the blood vessel wall and atherosclerotic plaque, and even reconstruct the lesion model, which is beneficial to the diagnosis and research of cardiovascular disease. But the challenge is that clinicians need to acquire more knowledge and applications of related interdisciplines and to understand the occurrence and development of diseases more accurately. In addition, the new technology faces the challenge of medical staff recognition.

\section{Fluorescence Lifetime Imaging}

Fluorescent molecules are excited by an external light source of a specific wavelength. Photon of this wavelength is absorbed by the molecule, resulting in the electronic transition from a ground state to an excited state. After transient vibration relaxation to the lowest excited electronic state, the excited molecule returns to the ground state in a radiative transition, emitting photon of longer wavelength, 
which is fluorescence. Fluorescence lifetime refers to the average residence time, the fluorescent molecules are in the excited state before they return to the ground state. It is not related to the concentration of fluorophore and the intensity of excitation, but is strongly influenced by the interaction with the microenvironment. FLIM is a technique based on measuring and analyzing the fluorescence lifetime distribution and variation in samples, and thus has the ability to probe the microenvironment.

Methods for measuring fluorescence lifetime in FLIM include frequency domain methods and time domain methods. ${ }^{8}$ Time domain methods have the advantages of fast imaging speed and high temporal resolution compared with frequency domain methods, and the fluorescence decay curves obtained from time domain methods directly reflects the fluorescence lifetime of the analyte. ${ }^{9}$ On the contrary, frequency domain methods require simpler equipment and is better than time domain methods in studies of samples with long-lived emission, ${ }^{10}$ and has higher application value under the condition of low imaging speed. Both time domain FLIM and frequency domain FLIM have been successfully used in the field of cell biology, analytical chemistry and clinical diagnosis to determine the distribution of physiological parameters of the sample. ${ }^{11}$

At present, the core components of FLIM are laser, detector and probe. Wide field FLIM requires low technology, and the system can be implemented by compact, portable, and low-cost all solid state diode pumped laser technology. It can be applied to situations requiring rapid imaging. ${ }^{12}$ Gated detection generally uses gated microchannel plate image intensifiers (MCP, Intensifier) or intensified CCD cameras to achieve wide field (full, field) fluorescence lifetime imaging of samples. With the development of TCSPC technology and solid-state ultrafast laser technology, the counting frequency and storage space of TCSPC technology have been greatly improved, and the conditions for fast measuring fluorescence lifetime have been achieved. ${ }^{13}$ By combining with confocal microscopy and multiphoton excitation fluorescence microscopy, the samples can be subjected to TCSPC-based multiphoton excitated fluorescence lifetime imaging. ${ }^{14,15}$ However, multi-spectral wide-field FLIM technology based on gated image intensifier can only obtain fluorescence lifetime images of up to two spectral bands at a time, while speed of the TCSPC-based multi-spectral resolution FLIM imaging is very low, which limits its application scope. In vivo FLIM imaging enhances sensitivity and selectivity of fluorescence diagnosis but requires a high level of equipment. Endoscopic FLIM with an exciting laser guided into the cavity through the optical fiber allows for the localization and diagnosis of the lesions in the cavity. In addition, when the fluorescence is not strong enough, an image intensifier can also be used to improve the resolving power. At present, the time scattering of optical fiber is not the key to develop an endoscopic FLIM system. ${ }^{16}$ However, it is imperative to address the development of low-cost, miniaturized, tunable ultra fast light sources, efficient coupling and transmission of exciting and emitted light through an endoscope and the elimination of complex autofluorescence signals present in most biological tissues.

\section{FLIM and Clinical Medicine}

Over the past 20 years, fluorescence measurement and imaging methods, often used to study biological events with various fluorescent probes including organic dyes, have been widely used in the life sciences due to their excellent performance and advantages mentioned above. FLIM provides more biochemical and medical diagnostic information for the identification of abnormal tissues in vivo compared with the traditional methods based on fluorescence intensity determination, and thus has the potential for wide application in clinical medical diagnosis.

\subsection{Cancer}

Cancer is the world's most deadly disease, and early diagnosis and location of cancer is the key to saving lives. The spontaneous fluorescence produced in cells and tissues can provide information on cell metabolism. FLIM based on auto-fluorescence can be used to rapidly detect the development of metabolic changes in precancerous lesions ${ }^{17}$ and provide the necessary chemical and anatomical information for pathologists and surgeons. FLIM has high sensitivity and specificity in the detection of cancer and precancerous lesions, allows accurate positioning of cancer tissue and provides a more accurate basis for surgical treatment. ${ }^{18}$ It was reported that the photosensitizer hematoporphyrin derivative $(\mathrm{HpD})$ has specific affinity for human malignant tumors and its 
fluorescence lifetime is shorter than that of normal tissue, and can thus distinguish normal and cancerous tissues. ${ }^{19,20}$ Furthermore, FLIM enables the early diagnosis of cancer and the measurement of living organisms. The auto-fluorescence characteristics of the cell cycle at different phases of the cancer cell response to changes in intracellular tryptophan and porphyrins, distinguishing normal cells from cancer cells. ${ }^{21}$

\subsection{Skin diseases}

Imaging technology has played an important role in the medical field since the discovery of X-rays. In the past 20 years, these techniques have been used for the detection of skin diseases. With the development of imaging technology and computer technology, research has accelerated, making clinical diagnosis easier. Multi-photon excitation (MPE) FLIM can significantly improve the imaging depth in tissues by up to $200 \mu \mathrm{m}$ in human skin autofluorescence lifetime imaging. The fluorescence lifetime distribution of the tissue which can be used to diagnose some skin diseases shows the changes in cell metabolism. ${ }^{22}$

As a noninvasive tool for the diagnosis of skin disease progression, the main areas of FLIM application are skin cancers related to melanin and pigmentation. Cubeddu et al. ${ }^{23}$ suggested that FLIM can recognize basal cell carcinoma (BCC) as the fluorescence lifetime is longer than that of normal tissue. Figure 1 shows fluorescence lifetime images of BCC. FLIM technology for biopsy and histological examination prior to resection can identify complete tumor margins and provide clinical guidance for skin lesions.

Rakesh et al. ${ }^{24}$ found that FLIM could be used to diagnose human benign dysplastic nevi (DN) and malignant nodular BCC. As shown in Figs. 2 and 3 , with increasing depth of the nevus, autofluorescence of the cells is becoming less obvious (only obvious within less than $60 \mu \mathrm{m}$ ). However, local destructive tumors which affect the normal skin structure can still be observed in the dermis of BCC.

Psoriasis is an autoimmune disease characterized by hyperkeratosis of the skin, excessive proliferation of the epidermis, accumulation of inflammatory cells, and increased vasodilatation of the dermal papilla, with increased metabolic activity in the epidermis and dermis. Kapsokalyvas et al. ${ }^{25}$ indicated that the fluorescence lifetime images showed morphological differences in fibroblasts between healthy skin and psoriasis, and the nicotinamide adenine dinucleotide (NADH) in psoriasis had longer life time compared with that in healthy skin, as shown in Fig. 4.

Fluorescence lifetime imaging techniques have been used in the in situ characterization of skin components. Dancik et al. ${ }^{26}$ imaged cell cultures of skin melanocyte cornification (dorsal and palmar forearm) from African and Asian volunteers with FLIM and identified the skin type and depth (granular layer and the underlying layer) by the melanin content and distribution. As shown in Fig. 5, the dorsal sides of volunteers in the two groups were darker than the palm sides. The spatial distribution of melanin is subject to further

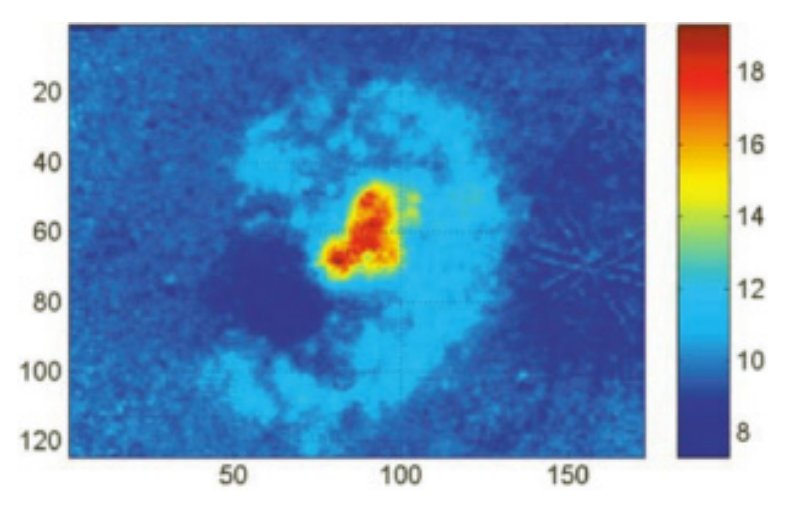

(a)

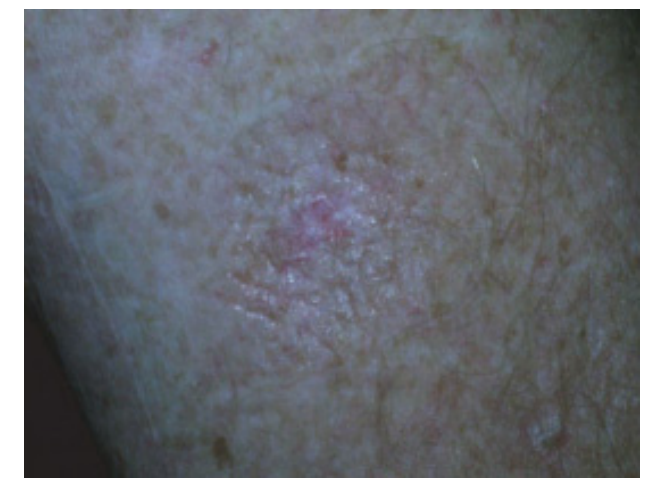

(b)

Fig. 1. (a) Fluorescence lifetime imaging of BBC of the skin, (b) skin color. ${ }^{23}$ (Permission has been granted by "IOP Publishing" through Copyright Clearance Center's RightsLink® service.) 


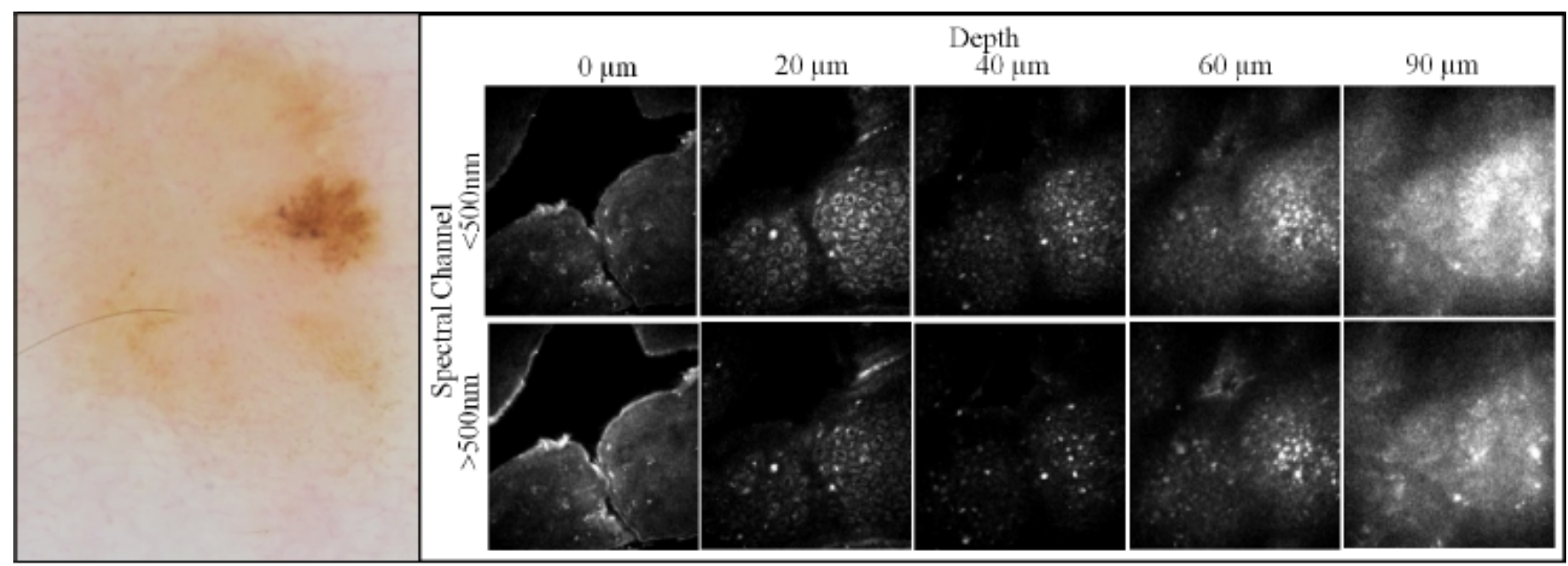

(a)

(b)

Fig. 2. (a) Nevus, (b) Fluorescence lifetime images at different depths, imaging field of view $177 \times 177 \mu \mathrm{m}^{2} .^{24}($ Permission has been granted by "SPIE".)

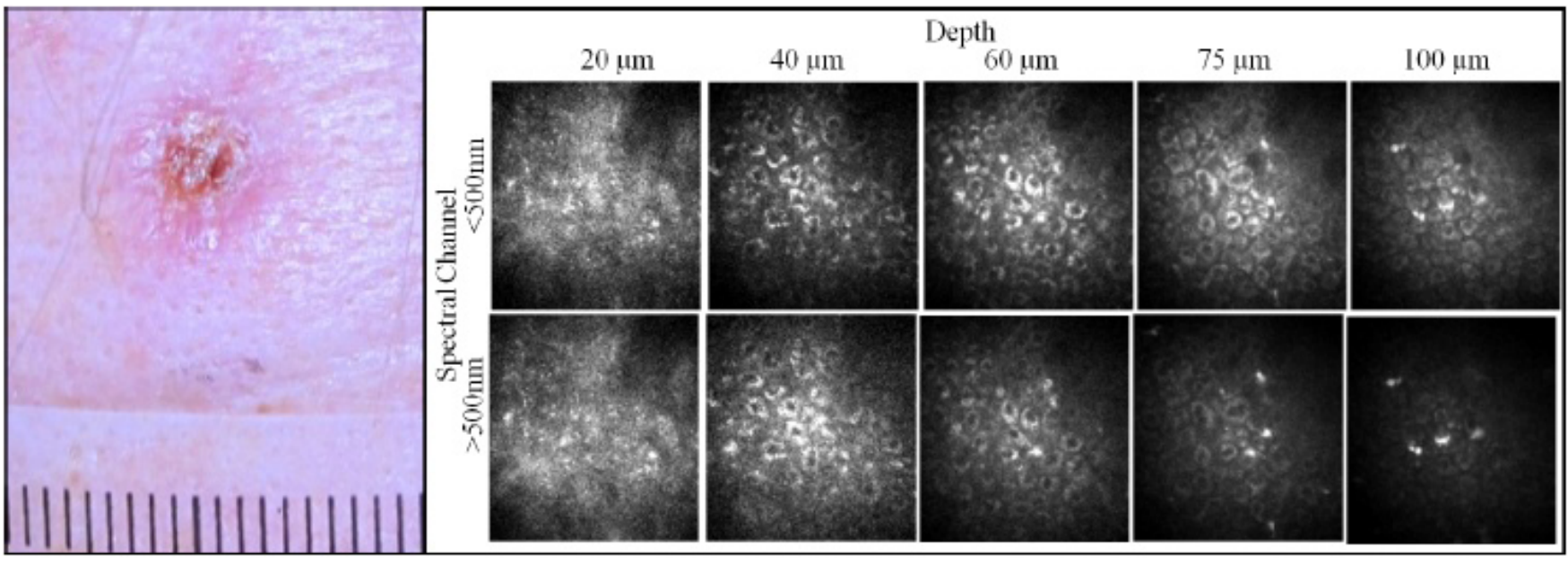

(a)

(b)

Fig. 3. (a) BCC, (b) Fluorescence lifetime images at different depths, imaging field of view $177 \times 177 \mu \mathrm{m}^{2} .^{24}(\mathrm{Permission}$ has been granted by "SPIE".)

investigation, including determination of melanin content in the skin at smaller gaps and depths. FLIM can be used to quantitatively assess skin characteristics in different parts of the body, and effectively identify skin diseases. ${ }^{27}$

Noninvasive FLIM combined with multi-photon tomography (MPT-FLIM), accurately determined the distribution and cell metabolism of mitochondria in inflammation, and is expected to be used to study the pathophysiological mechanism of inflammatory skin disease and in early clinical diagnosis and treatment. ${ }^{28}$ In addition, MPT-FLIM can accurately identify the edge of tumors, providing the clinical basis for surgical treatment.

\subsection{Brain diseases}

The brain is the most sophisticated organ in the body and is involved in cognition and memory. Although FLIM has characteristics of noninvasive, nontoxic and nonionizing radiation, ${ }^{29}$ the direct access to the brain is always a problem. Therefore, 


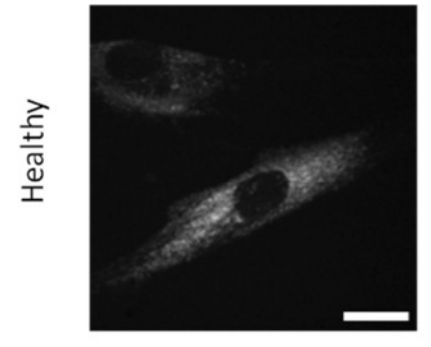

(a)

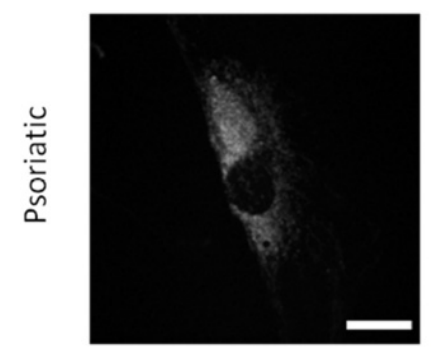

(c)

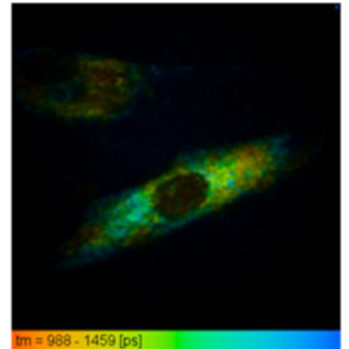

(b)

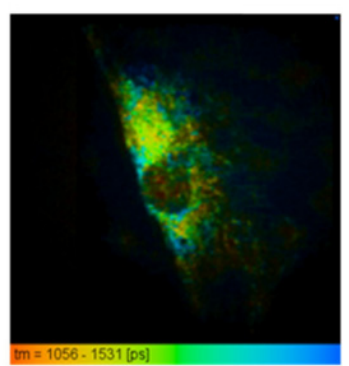

(d)
Fig. 4. (a) Healthy fibroblast fluorescence intensity image, (b) fluorescence lifetime color coded image; (c) Psoriatic fibroblast fluorescence intensity image, (d) fluorescence lifetime color coded image $($ scale bar $=20 \mu \mathrm{m}){ }^{25}$ (Permission has been granted by "SPIE".)

(a)
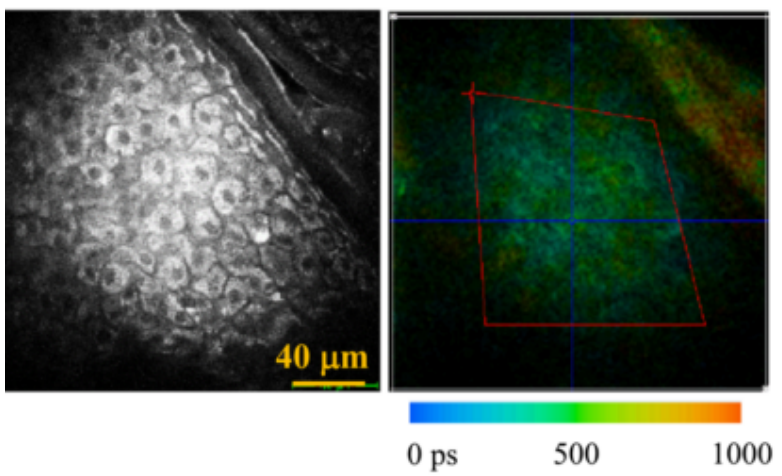

(c)

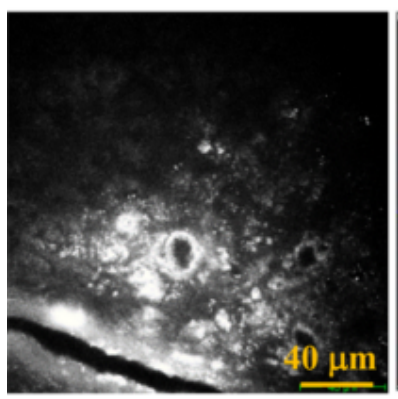

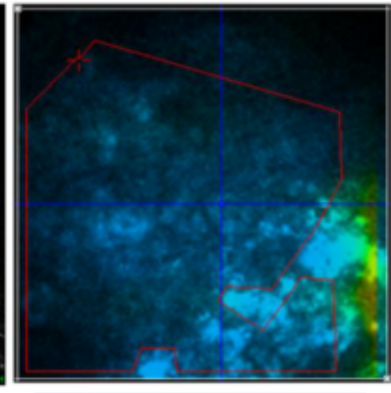

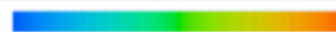

applications in brain are different from most other organs yet examined by FLIM (skin, Mucosa, eye, teeth. .). In vivo or in situ examination of the brain requires craniotomy and durotomy and is therefore limited to neurosurgical procedures only, rather than for purely diagnostic (noninvasive) purposes.

Neural circuits are the structural basis of brain functional activities. The study of neural circuits is of great significance in the study of brain diseases and brain injury. ${ }^{30}$ In the study of cranial nerve circuits, it is helpful to study the function and life span of nerve cells by measuring the ion concentration in these cells. According to Gensch et al. ${ }^{31}$ calcium as a messenger is commonly found in all cell types, and FLIM is able to determine brain function by measuring changes in calcium concentrations in living cells and tissues.

High-resolution MPT-FLIM can distinguish glioma from normal brain tissue. At present, MPT scanning is used in experimental studies and for clinical diagnosis in dermatology, and FLIM has been used during brain tumor surgery ${ }^{32}$. FLIM measures cell density in tumor cells in a few seconds

(b)

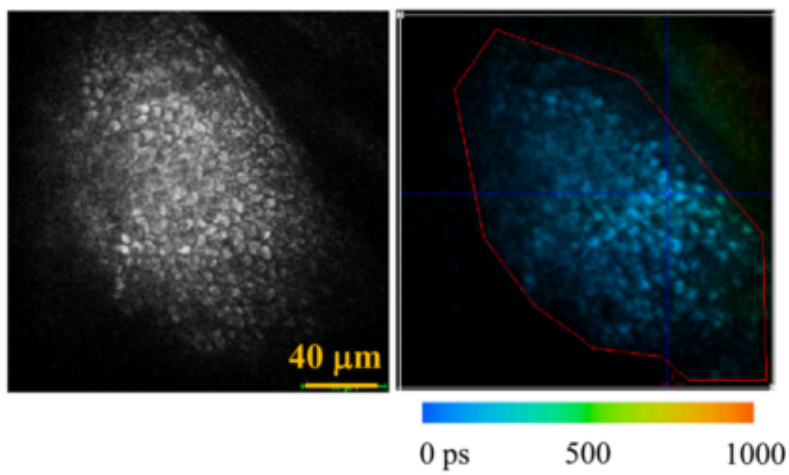

(d)

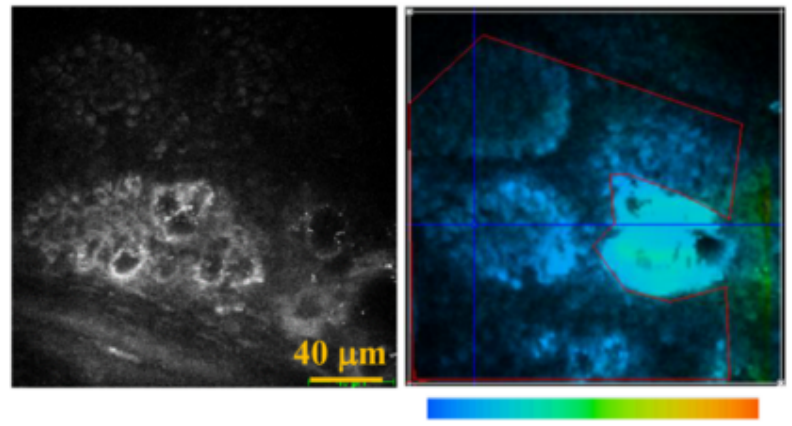

Fig. 5. Fluorescence lifetime imaging of the dorsal and volar forearm. Asian volunteer's (a) stratum granulosum and (b) stratum basale. African volunteer's (c) stratum granulosum and (d) stratum basale. ${ }^{26}$ (Permission has been granted by "SPIE".) 

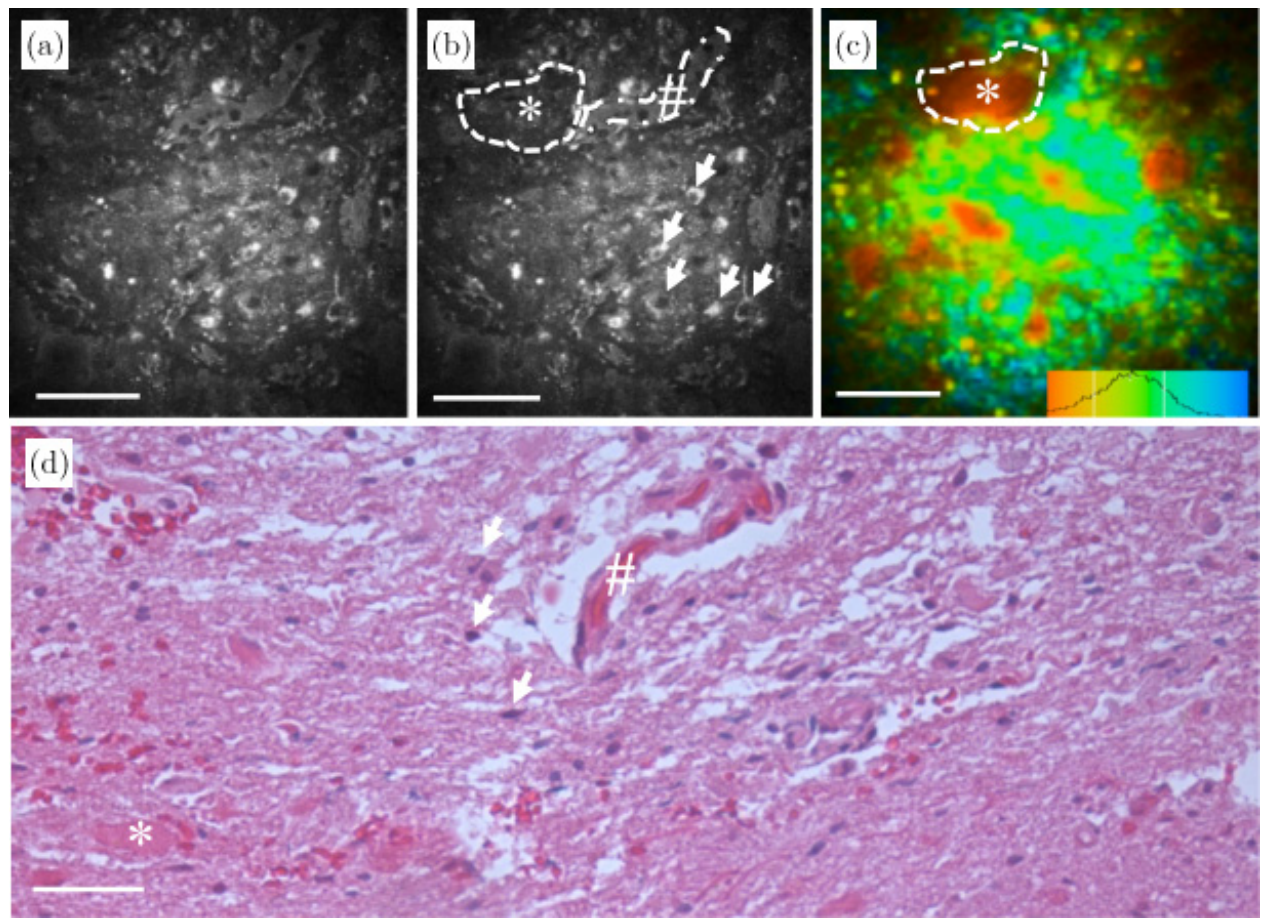

Notes: Necrosis is marked with "**".

Microvascular proliferation is marked with "\#".

Fig. 6. Multiphoton intensity and fluorescence lifetime imaging of human glioma tissue ex vivo. (a) and (b) fluorescence intensity images, (c) fluorescent lifetime images, (d) conventional histology of the tumor (HE (hematoxylin-eosin) staining of a region resembling the area examined by MPT), examples of cell pleomorphism are marked with white arrows. The white bars correspond to a distance of

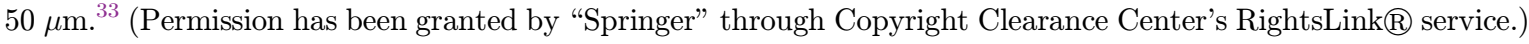

at the cellular and subcellular levels without tissue treatment or staining to identify glioma tissue, ${ }^{33,34}$ as shown in Fig. 6. In order to reduce the loss of function of healthy brain tissue, radical resection of the lesion should be performed by neurosurgeons and FLIM is capable of improving the accuracy of surgery.

Timm et $a l^{35}$ found that dysfunction of microtubule affinity regulating kinase (MARK) is associated with cancer or brain disease (absence of cerebrospinal malformation and Alzheimer's disease $[\mathrm{AD}]$ ), as shown in Fig. 7, and biosensor inhibitors based on FLIM have the potential to treat AD.

At present, use of FLIM as a quantitative calibration technique in vivo is mainly limited to the acquisition speed of $3 \mathrm{D}$ and $4 \mathrm{D}$ imaging. With time, the development of 3D in vivo FLIM will achieve the quantitative diagnosis of neurological dysfunction or inflammation. ${ }^{36}$

\subsection{Eye diseases}

FLIM is a high-sensitivity tool for pathology research and clinical diagnosis in ophthalmology.
The fluorescence lifetime imaging ophthalmoscope (FLIO) is a new technique for measuring the temporal resolution based on auto-fluorescence generated by endogenous fluorophores. Dysli et al. ${ }^{37}$ investigated the potential prognostic markers of Stargardt disease (STGD) progression using FLIO. As shown in Fig. 8, a new lesion in the patient with a shorter auto-fluorescence lifetime was evidence of the accumulation of retinoic acid in the visual cycle. After a certain period of time, with the accumulation of lipofuscin, the fluorescence lifetime of the above-mentioned lesion was longer. The FLIO was used to predict the progression of STGD, and to evaluate the clinical therapeutic effect in STGD.

Dysli et $a l .{ }^{38}$ also studied the relationship between the fluorescence lifetime and co-occlusion of the central retinal artery and branch retinal artery by FLIO and found that the retinal artery occlusion in the acute phase leads to a longer fluorescence lifetime. Due to the increase in scattering in the ischemic area of the retina, the average lifetime was longer than that of the surrounding retinal tissue (including the fovea). In addition, patients with 


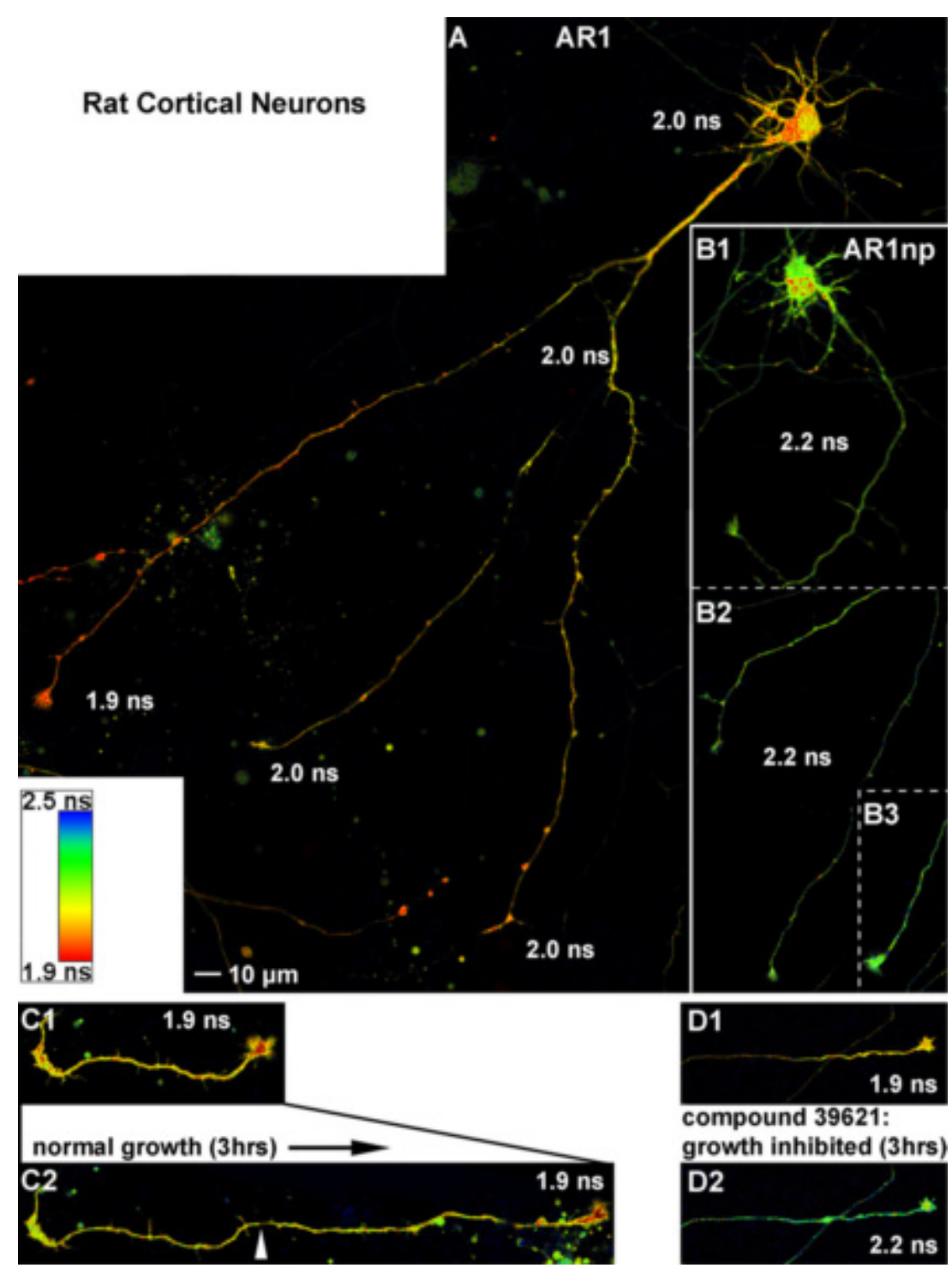

Fig. 7. Fluorescence lifetime images of AD mouse nerve growth. ${ }^{35}$ (Permission has been granted by "The American Society for Biochemistry and Molecular Biology" through Copyright Clearance Center's RightsLink® service.

increased flavin adenine dinucleotide, accumulation of advanced glycation end products (AGE), and decreased nicotinamide adenine dinucleotide in the fundus were found to have diabetic eyes using FLIM. Both can be used as indicators of metabolic changes in diabetic patients without diabetic retinopathy. ${ }^{39}$ Therefore, FLIO used to diagnose acute ischemic retinal injury, can measure the decay time of endogenous retinal fluorescence, and provide new clues for a better understanding of ischemic retinal disease.

FLIM has been used in the diagnosis of corneal epithelial wound healing. Early diagnosis is critical to prevent corneal epithelial wound healing infection, and to avoid loss of vision by continuous destruction of the corneal stroma. Gehlsen et al. ${ }^{40}$ detected the auto-fluorescence of the cell metabolism factor $\mathrm{NAD}(\mathrm{P}) \mathrm{H}$ in vivo using FLIM to evaluate the healing of wound epithelial cells, as shown in Fig. 9. The metabolism of substances in preclinical and clinical trials has become an indispensable diagnostic index in the clinic.

\subsection{Oral and bone diseases}

FLIM has very good contrast in different tissues in different states, such as collagen and elastin. The combination of endoscopic techniques and FLIM enable the detection and diagnosis of cancer and precancerous lesions in oral tissue and can be used for real-time monitoring of minimally invasive 


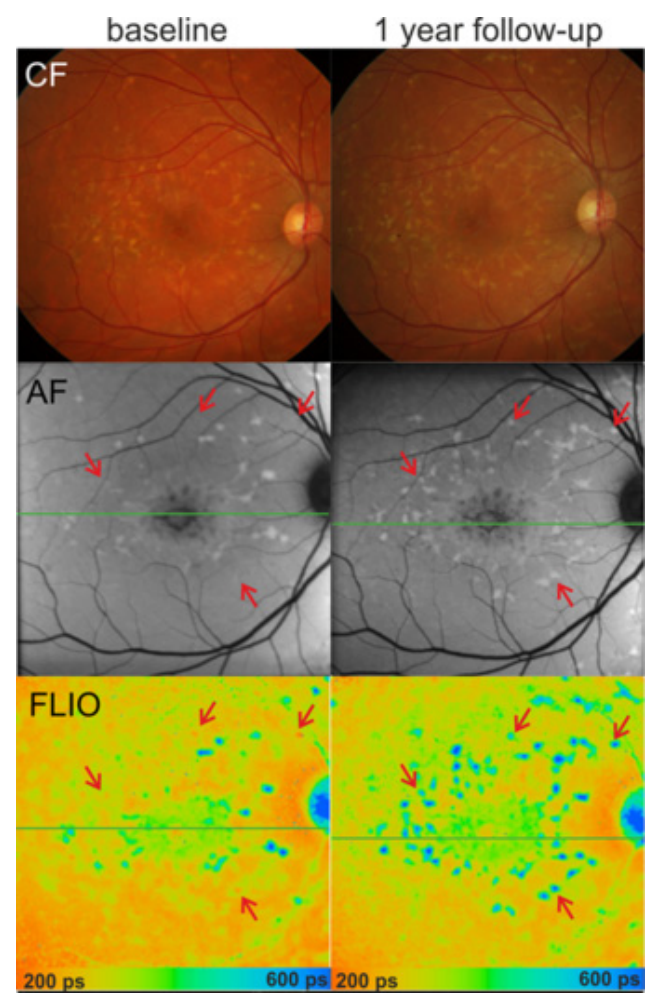

Fig. 8. STGD progression within one year. Color fundus (CFs), fundus AF, and FLIO (long spectral channel) with indicated lines of the optical coherence tomography scan (OCT) (baseline, left) A one year follow-up examination (right) shows clear disease progression with accumulation of hyperfluorescent flecks. The red arrows mark areas with short fluorescence lifetimes that converted to long lifetimes within one year. ${ }^{37}$ (Permission has been granted by "The Association for Research in Vision and Ophthalmology" through Creative Commons Attribution, http://creativecommons.org/licenses/by-nc-nd/4.0.) surgery. ${ }^{41}$ As shown in Fig. 10, a mobile portable fiber optic endoscope for flexible monitoring and guidance of surgery are used, and during FLIM for the diagnosis of oral cancer, a decrease in fluorescence intensity of diseased tissue was found compared to the surrounding normal tissue. ${ }^{42}$ Fatakdawala et al. ${ }^{43}$ reported that multimodal system joint diagnosis using a combination of FLIM, ultrasound backscatter microscopy (UBM) and photoacoustic imaging (PAI) can complement each other in the diagnosis of oral cancer in vivo.

FLIM based on gated detection was used in the diagnosis of bone and tooth disorders for noninvasive imaging of normal teeth and caries in the late 20th century. ${ }^{44,45}$ Lin et al. ${ }^{46}$ used multimodal (second harmonic, auto-fluorescence, and fluorescence lifetime) method with unlabeled analysis to differentiate the morphological characteristics of dentin and enamel (Fig. 11). Dental caries damages the structure of dental tissues by degrading the organic matrix. Auto-fluorescence lifetime was shown to decrease from the dentin and enamel to the caries area, which was related to the degree of demineralization and consistent with the results of micro-computed tomography, indicating that two-photon excited FLIM could be used to diagnose and monitor dental caries.

\subsection{Vascular and cavity organ diseases}

Conventional light microscopy plays an important role in the diagnosis of most liver diseases.
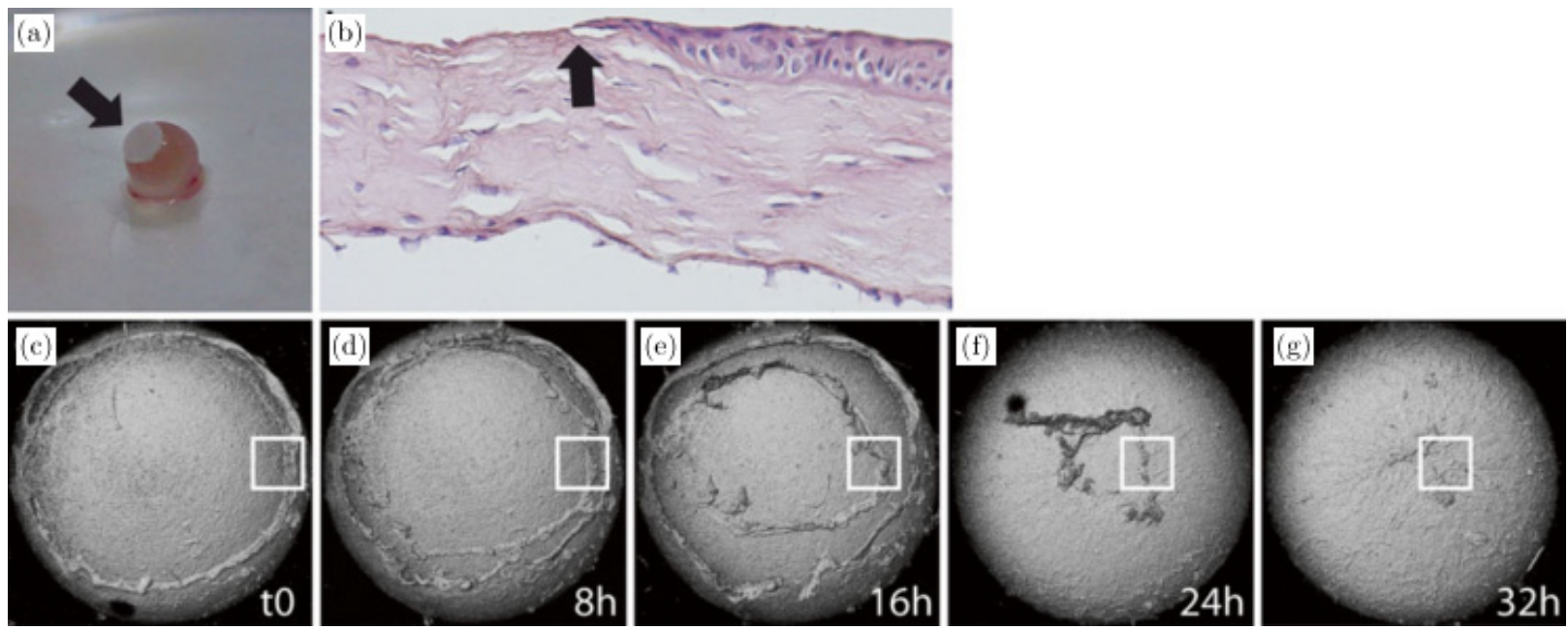

Fig. 9. Explanted murine eye model. (a) Explanted eye with a NaOH-containing filter paper (arrow) placed on the cornea. (b) Section through the edge of the erosion. At the leading edge, epithelial cells are elongated (arrow) and stretched towards the central cornea. (c)-(g). OCT imaging of the cornea demonstrates the time course of epithelial wound healing. ${ }^{40}$ (Permission has been

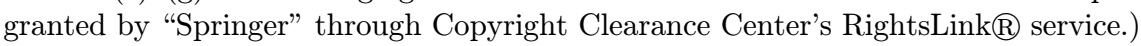



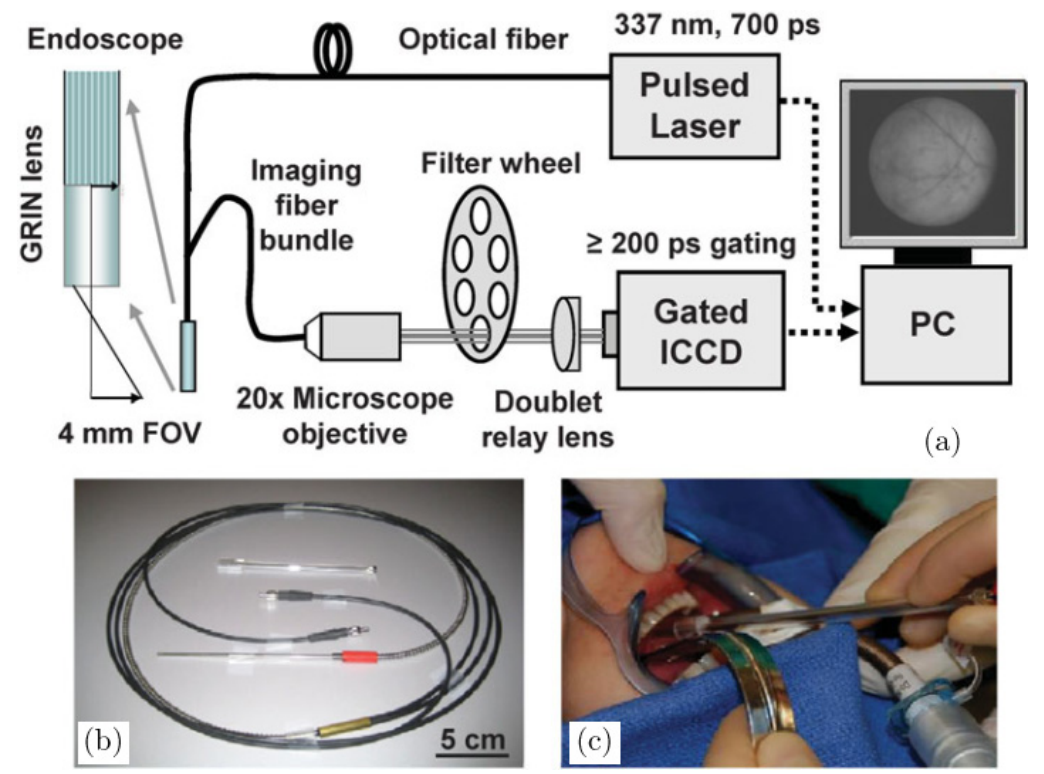

Fig. 10. Fluorescence lifetime microscopic imaging system for monitoring oral cancer. (a) Schematic diagram of FLIM endoscope, GRIN: gradient refractive index, FOV: field of view, ICCD: intensified charge-coupled device, PC: personal computer, (b) Semi flexible endoscope for surgery, (c) FLIM probe for oral cavity. ${ }^{42}$ (Permission has been granted by "Cambridge University Press" through Copyright Clearance Center's RightsLink® service.)

FLIM can image and quantify cell morphology and probe the liver microenvironment without conventional biopsy or fluorescent dye. Wang et al. ${ }^{47}$ believe that FLIM will be used from the laboratory to

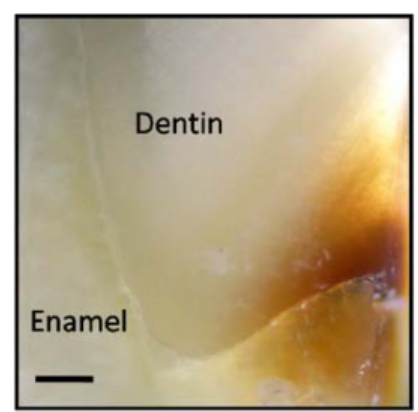

(a) the clinic for real-time histology and dynamic monitoring of human liver disease in the near future. In 1999, Mizeret et $a ._{.}^{48}$ developed the video frequency domain FLIM in vivo and applied it in

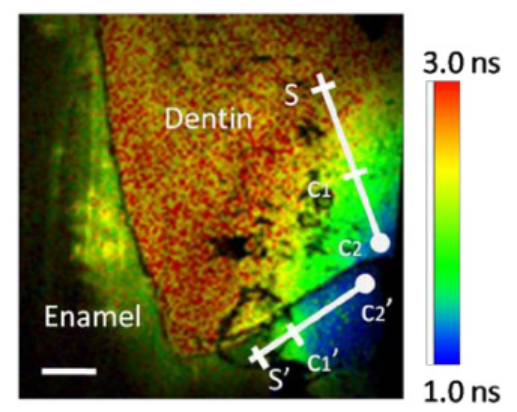

(b)

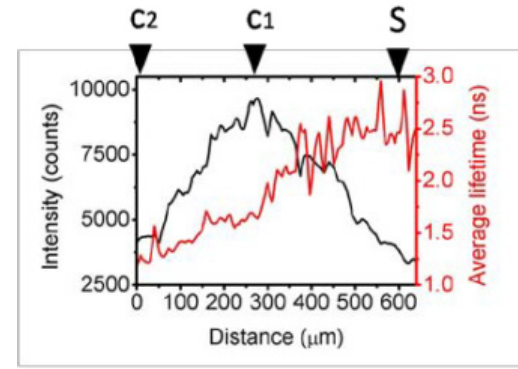

(c)

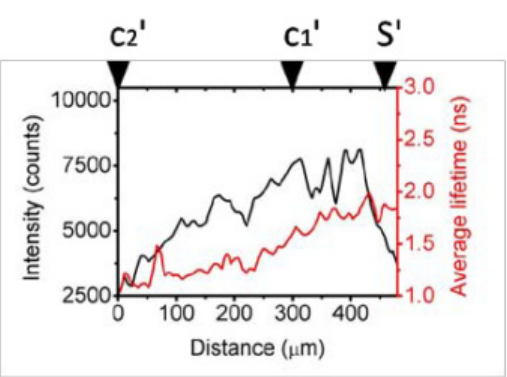

(d)

Fig. 11. (a) Dental caries image, (b). Dental caries FLIM image, scale bar $=200 \mu \mathrm{m}$, the decrease in lifetime in the caries area, black represents enamel, red represents dentine. The average lifetime section line: (c) dentine and (d) enamel. ${ }^{46}$ (Permission has been granted by "Biomedical Optics Express".) 


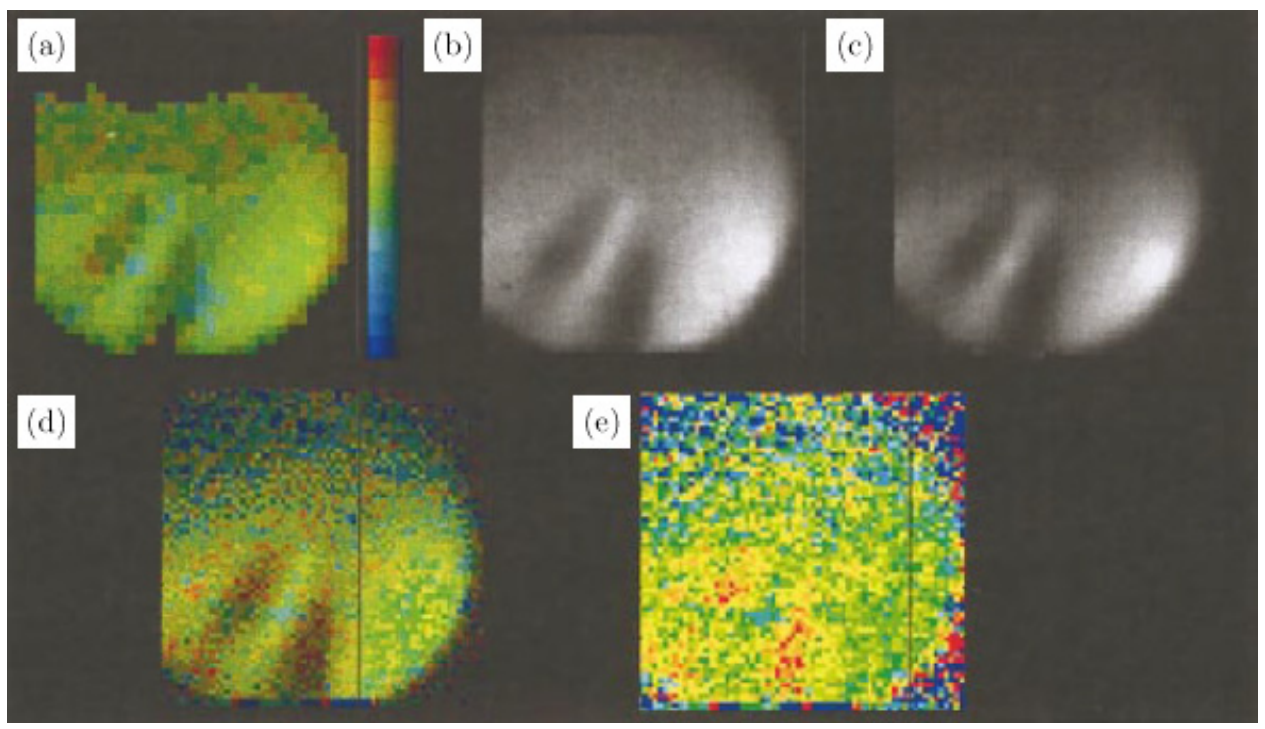

Fig. 12. Fluorescence lifetime imaging of anterior basal bronchus endoscopy. Blue-green represents normal tissue. ${ }^{48}$ (a) Apparent fluorescence lifetime image calculated during the endoscopic examination, based on the demodulation; (b) and (c) fluorescence and backscattered intensity images; (d) apparent fluorescence lifetime image recalculated after the examination; (e) apparent fluorescence lifetime image recalculated after the examination without intensity weighting (Permission has been granted by "AIP Publishing LLC" through Copyright Clearance Center's RightsLink® service.)

the field of medical diagnosis (Fig. 12), especially in gastric cancer and esophageal cancer. Compared with in vitro diagnostic methods (such as X-ray imaging and nuclear magnetic resonance imaging), FLIM can identify lesions not unrecognized in vitro.

Gorpas et al. $^{49}$ believed that atherosclerotic coronary vessels were involved in different pathological changes in blood vessel walls, which appeared to be a sub-type of various plaques. FLIM data identified different types of atherosclerotic plaques as a clinical diagnostic method. In recent years, research has focused on the combination of FLIM and traditional intravascular ultrasound (IVUS) to obtain information on atherosclerotic plaque shape. ${ }^{49-51} \mathrm{Bec}$

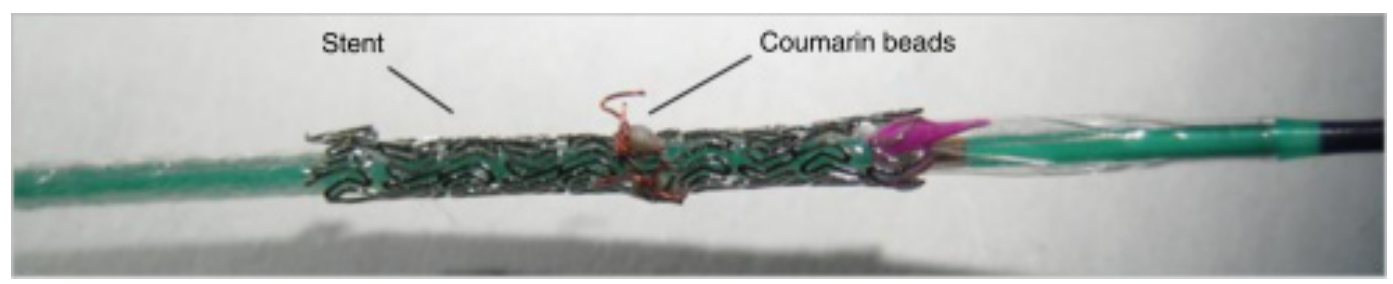

(a)

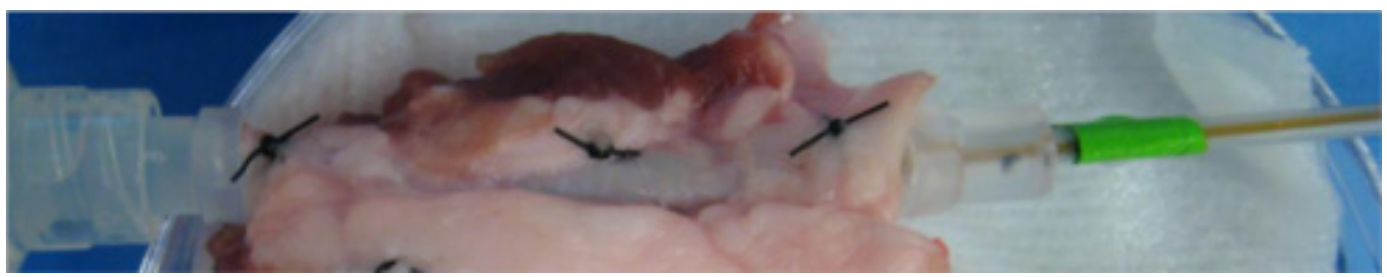

(b)

Fig. 13. IVUS-FLIM coregistration: evaluation of a coronary artery phantom. (a) Stent before deployment, (b) stent inserted in pig coronary artery; (c) open-loop intensity image; (d) folded open intensity lifetime image with intensity threshold; (e) IVUS image cross-section; and (f) reconstructed. 3D FLIM image of the coronary lumen. ${ }^{50}$ (Permission has been granted by "SPIE".) 


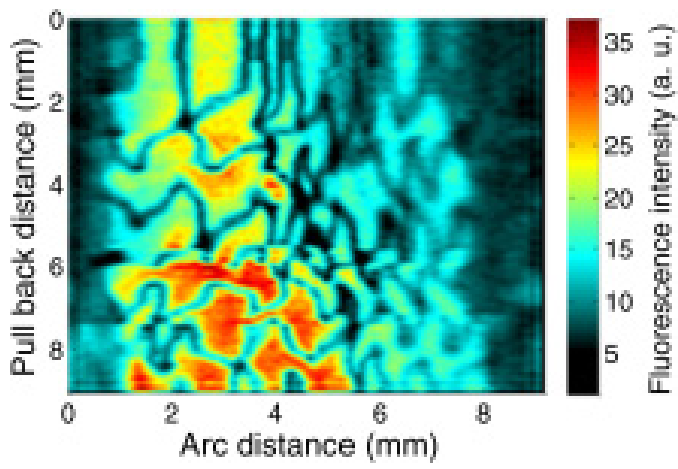

(c)

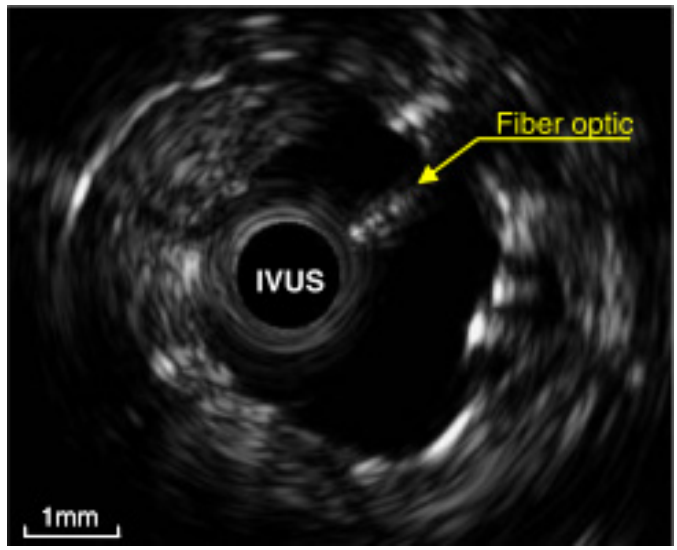

(e)

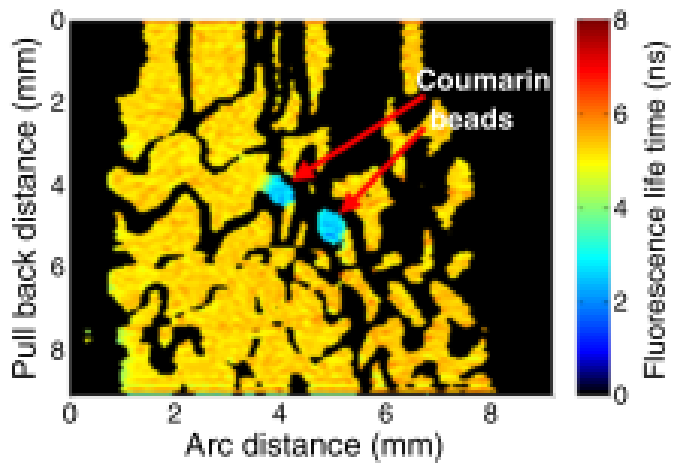

(d)

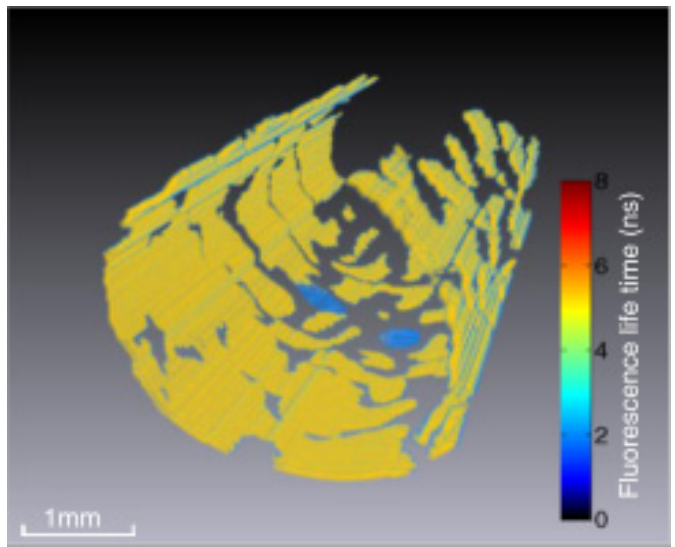

(f)

Fig. 13. (Continued)

et $a l .{ }^{50}$ combined FLIM with IVUS in a porcine coronary artery model, and suggested that the use of FLIM was feasible for diagnosing coronary atherosclerotic plaques in vivo by synthesizing biochemical and morphological information, as shown in Fig. 13.

The FLIM method can determine the ratio of lipid content in the collagen of fibrous caps of atherosclerotic plaques and assess the risk of plaque rupture. The collagen lipid ratio has been proved to be an important parameter for evaluating the structural integrity of fibrous caps. Phipps et al. ${ }^{52}$ showed that FLIM could distinguish between collagenous and lipid-rich regions of the carotid plaque area without the assistance of fluorescence intensity or a contrast agent and assess the degree of plaque rupture by collagen auto-fluorescence decay, as shown in Fig. 14.

\subsection{Drug evaluation}

In the treatment of diseases, the interaction between drug molecules and pathological tissue molecules will change the microenvironment of cells. Therefore, the effect of drug treatment can be evaluated by studying the microenvironment of cells and tissues. ${ }^{53-55}$ The specificity of FLIM can quantitatively detect a variety of exogenous chemicals, nanoparticles added to the skin and endogenous fluorescent substances in the skin. Quantitative imaging of coexisting drugs and their metabolites, skin $\mathrm{pH}$, nano-zinc oxide penetration of the skin, deep tissue liposomal administration and skin aging can be observed in various skin diseases, ${ }^{56}$ while, the efficacy is evaluated by the changes in metabolism of metal nanoparticles penetrating human skin. ${ }^{57}$ 


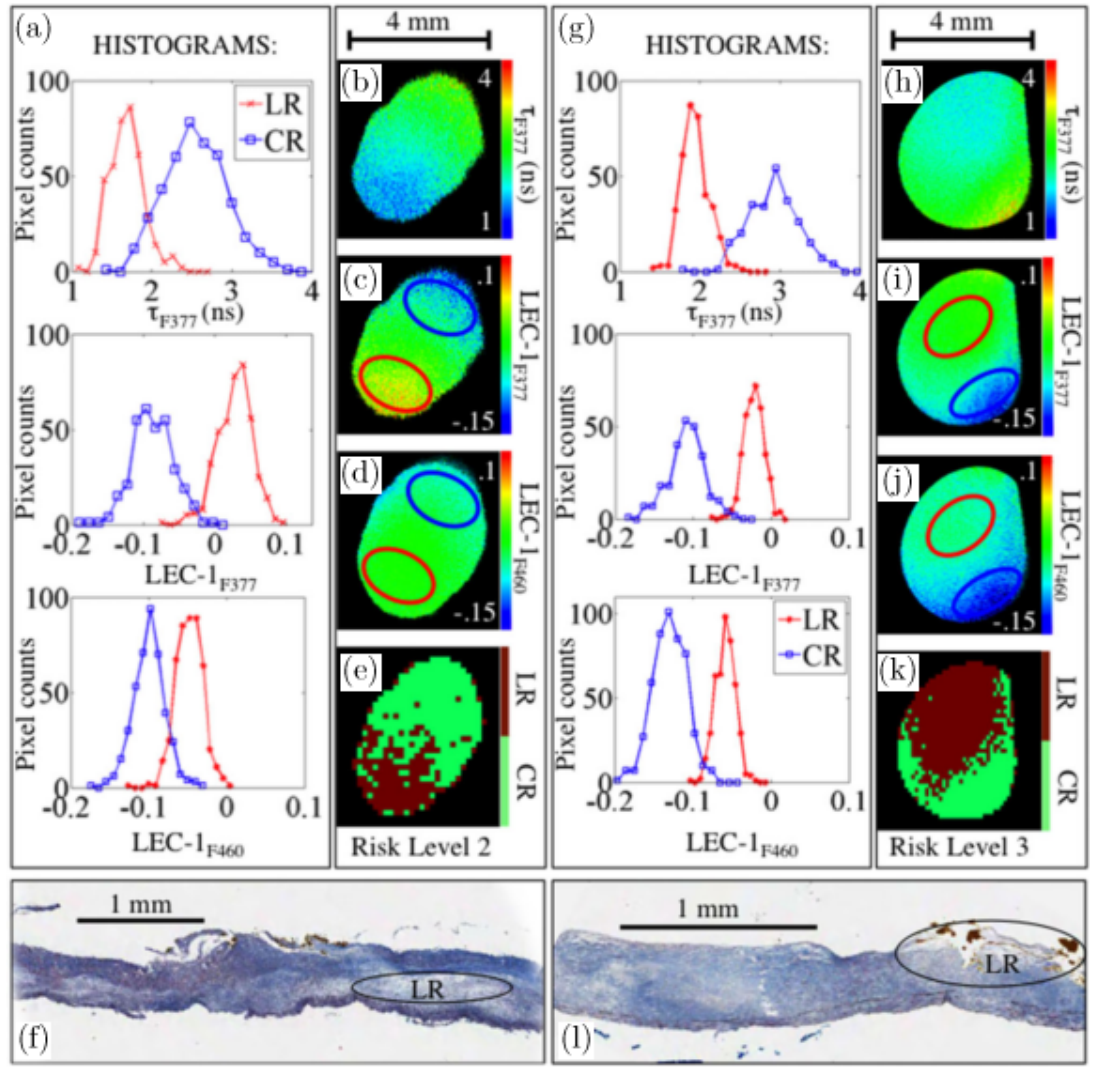

Fig. 14. Fluorescence lifetime images of carotid plaque region. (a) and (g): Histograms from images in (b)-(d), and (h)-(j), respectively. Blue circle: collagen-rich (CR) region, red circle: lipid-rich (LR) region. (e) and (k): Classification of entire image. (f) and (l): Trichrome stained tissue sections from FLIM data shown in (a)-(e) and (g)-(k), respectively. ${ }^{52}$ (Permission has been granted by "John Wiley and Sons" through Copyright Clearance Center's RightsLink@ service.)

(a)

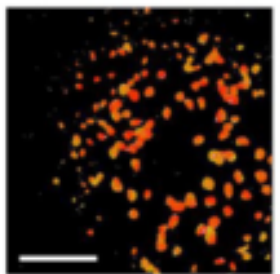

$\operatorname{DXR}(-)$

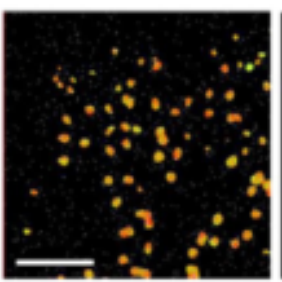

$\operatorname{DXR}(+) 3 \mathrm{hr}$

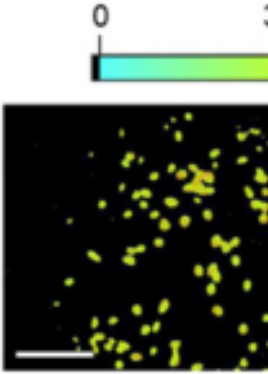

$\operatorname{DXR}(+) 6 \mathrm{hr}$
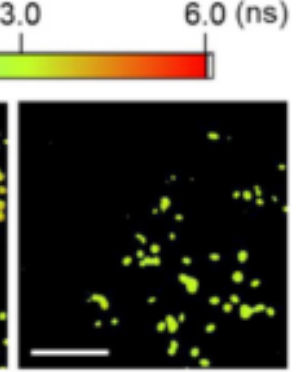

$\operatorname{DXR}(+) 12 \mathrm{hr}$

(b)

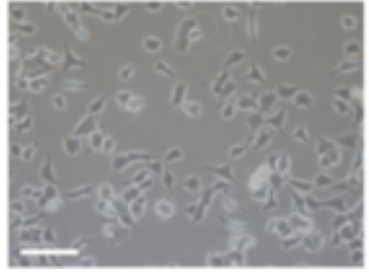

Fig. 15. (a) The FLIM images of the nucleus in RDES cells labeled with SYTO 13 before and after treatment with $500 \mathrm{nM}$ of doxorubicin (DXR). (b) A phase-contrast image of RDES cells without treatment with DXR. Scale bar represents $100 \mu \mathrm{m}{ }^{58}$ (Permission has been granted by "IOP Publishing" through Copyright Clearance Center's RightsLink $($ service.) 
Table 1. Retrieval results of fluorescence lifetime imaging in tissues and organs.

\begin{tabular}{lccccccccc}
\hline & \multicolumn{8}{c}{ Fluorescence lifetime imaging } \\
\cline { 2 - 10 } Key words & Cancer & Skin & Dental & Oral & Blood vessel & Cardiovascular & Eye & Brain & Bone \\
\hline Items & 412 & 86 & 14 & 43 & 47 & 62 & 43 & 116 & 18 \\
\hline
\end{tabular}

FLIM can evaluate the efficacy of anticancer drugs. Kawanabe et al. ${ }^{58}$ (Fig. 15) reported that antitumor efficacy can be evaluated by the degree of decline in the fluorescence lifetime of cells treated with different drugs and FLIM provides useful information at the early stages of apoptosis and provides a clinical guide on the efficacy of anticancer drugs.

\section{Summary}

Fluorescence lifetime imaging technology, which is sensitive to the microenvironment of fluorophores and can probe the changes and distribution of physiological parameters in the sample, has been successfully used in cell biology, analytical chemistry, clinical diagnosis and other fields. FLIM can determine the accurate location of cancer tissue and provide a more accurate basis for surgical treatment with high sensitivity and specificity. In addition, as can be seen from the annex, the technical level of each FLIM equipment is not very different. The gap is mainly in the part of the combination device, because the diagnosis and treatment of the diseases of different tissues and organs are different. Among them, the most advanced is the combination device of cavity organs, such as endoscopy and intravascular ultrasound. The development of these two devices has been relatively mature, and has also been widely used in clinical. The application of fluorescence lifetime imaging in various organ tissues is retrieved on PubMed (https://www.ncbi. nlm.nih.gov/pubmed), unlimited time, as shown in Table 1. There are so many studies of cancer, more research on the cardiovascular system and brain, which may be relevant to the research hotspot. Retrieval of brain applications within five years, the items are 63 , while neuroscience research is popular in recent years.

In general, current FLIM diagnosis and research in clinical medicine is focused on common diseases, especially tumors. At the same time, there are few fluorescence lifetime imaging diagnostic devices in clinic. In previous studies, we found that the research scope of tissues and organs is narrow and lack of systematic data collection. Device imaging speed and field of view also affect its application and a recognition of new technologies by clinicians is also important factor. For the future, we believe that structural remodeling will be gradually developed in the field of fluorescence lifetime imaging, and thus will be better utilized in clinic. Relevant researches have been done, such as Rinnenthal et al. and Julin et $a l^{36,50}$ In recent years, with the development of precision medicine and superresolution technology, more and more researches have been done on the combination of superresolution and FLIM. At the same time, better laser light sources, such as fiber laser $(350 \mathrm{~nm}, 1 \mathrm{MHZ}$ repetition rate), guarantee scanning speed and imaging quality and better detection depth improves the temporal and spatial resolution of surface diagnostics. ${ }^{9}$ We believe that with its advantages and characteristics, FLIM will have a profound impact on the treatment of traditional diseases, and has a wide range of clinical applications.

\section{Acknowledgments}

The project was funded by the Science and Technology Planning Fundamental Research Project of Shenzhen (No. JCYJ20150324140036853), National Natural Science Foundation of China (No. 61378091), Ningbo Natural Science Foundation Project (No. 2016A610032) and the Central University Basic Scientific Research Business Expenses Project (No. NSIY051405). Zhanwen Wang, Yanping Zheng and Deqiang Zhao contributed equally to the work.

\section{References}

1. M. R. Hight et al., "Multispectral fluorescence imaging to assess $\mathrm{pH}$ in biological specimens," J. Biomed. Opt. 16, 016007 (2011).

2. P. P. Hsu, D. M. Sabatini, "Cancer cell metabolism: Warburg and beyond," Cell. 134 703-707 (2008). 
3. C. Stringari, R. A. Edwards, K. T. Pate et al., "Metabolic trajectory of cellular differentiation in small intestine by phasor fluorescence lifetime microscopy of NADH," Sci. Rep. 2, 00568 (2012).

4. R. Yasuda, C. D. Harvey, H. N. Zhong et al., "Supersensitive Ras activation in dendrites and spines revealed by two-photon fluorescence lifetime imaging," Nat. Neurosci. 9, 283-291 (2006).

5. T. Nakabayashi, S. Oshita, R. Sumikawa et al., "pH dependence of the fluorescence lifetime of enhanced yellow fluorescent protein in solution and cells," J. Photochem. Photobiol. A-Chem. 235, 65-71 (2012).

6. W. Becker, "Fluorescence lifetime imagingtechniques and applications," J. Microsc. 247, 119-136 (2012).

7. A. Pliss, X. Peng et al., "Single cell assay for molecular diagnostics and medicine: Monitoring intracellular concentrations of macromolecules by two-photon fluorescence lifetime imaging," Theranostics, 5, 919-930 (2015).

8. E. B. van Munster, T. W. J. Gadella, "Fluorescence lifetime imaging microscopy (FLIM)," Adv. Biochem. Eng. Biotechnol. 95, 143-175 (2005).

9. L. Marcu, "Fluorescence lifetime techniques in medical applications," Ann. Biomed. Eng. 40, 304-331 (2012).

10. K. Suhling, P. M. W. French, D. Phillips, "Timeresolved fluorescence microscopy," Photochem. Photobiol. Sci. 4, 13-22 (2005).

11. L. K. Seah, P. Wang, V. M. Murukeshan et al., "Application of fluorescence lifetime imaging (FLIM) in latent finger mark detection," Forensic. Sci. Int. 160, 109-114 (2006).

12. S. Wachsmann-Hogiu, J. Y. Hwang et al., "Widefield two-photon microscopy: Features and advantages for biomedical applications," Proc. SPIE 6441, 64411B (2007).

13. W. Becker, A. Bergmann et al., "High resolution TCSPC lifetime imaging," Proc. SPIE, 4963, 175-184 (2003).

14. L. Liu, Z. Lin, J. Qu et al., "Multifocal two-photon excitation fluorescence sampling imaging combining lifetime and spectrum resolutions," Proc. SPIE 6047, 60472T (2005).

15. L. Liu, J. Qu, L. Wang et al., "Five-dimensional multifocal multiphoton microscopy," Proc. SPIE 6534, 65341Q (2007)

16. L. Liu, Z. Lin, J. Qu et al., "Fluorescence lifetime imaging and its biomedical applications," J. Shenzhen Univ. Sci. Eng. 22, 133-141 (2005).

17. P. P. Provenzano, K. W. Eliceiri, P. J. Keely, "Multiphoton microscopy and fluorescence lifetime imaging microscopy (FLIM) to monitor metastasis and the tumor microenvironment," Clin. Exp. Metastasis 26, 357-370 (2009).

18. A. J. Sherman, A. Papour, S. Bhargava et al., "Normalized fluorescence lifetime imaging for tumor identification and margin delineation," Proc. SPIE, 8572, 85721H (2013).

19. J. R. Lakowicz, K. W. Berndt, "Lifetime-selective fluorescence imaging using an Rf phase-sensitive camera," Rev. Sci. Instrum. 62, 1727-1734 (1991).

20. R. Cubeddu, G. Canti, P. Taroni et al., "Time-gated fluorescence imaging for the diagnosis of tumors in a murine model," Photochem. Photobiol. 57, 480-485 (1993).

21. X. G. Lin, Y. J. Pan, Y. C. Guo, "The study of cervical cancer cells model based on UV absorption spectrum," Spectrosc. Spect. Anal. 29, 1328-1331 (2009).

22. B. R. Masters, P. T. So, E. Gratton, "Multiphoton excitation fluorescence microscopy and spectroscopy of in vivo human skin," Biophys. J. 72, 2405-2412 (1997).

23. R. Cubeddu, D. Comelli, C. D'Andrea et al., "Timeresolved fluorescence imaging in biology and medicine," J. Phys. D. Appl. Phys. 35, 61-76 (2002).

24. R. Patalay, C. Talbot, I. Munro et al., "Fluorescence lifetime imaging of skin cancer," Proc. SPIE $\mathbf{7 8 8 3}$, 78830A (2011).

25. D. Kapsokalyvas, V. Barygina, R. Cicchi et al., "Evaluation of the oxidative stress of psoriatic fibroblasts based on spectral two-photon fluorescence lifetime imaging," Proc. SPIE 8588, 85882D (2013).

26. Y. Dancik, A. Favre, C. J. Loy et al., "Use of multiphoton tomography and fluorescence lifetime imaging to investigate skin pigmentation in vivo," J. Biomed. Opt. 18, 026022 (2013).

27. E. Benati, V. Bellini, S. Borsari et al., "Quantitative evaluation of healthy epidermis by means of multiphoton microscopy and fluorescence lifetime imaging microscopy," Skin Res. Technol. 17, 295-303 (2011).

28. V. Huck, C. Gorzelanny, K. Thomas et al., "From morphology to biochemical state - intravital multiphoton fluorescence lifetime imaging of inflamed human skin," Sci. Rep. 6, 22789 (2016).

29. A. Schweitzer, H. Eipel, C. Cremer, "Rapid image acquisition in multi-photon excitation fluorescence microscopy," Optik 115, 115-120 (2004).

30. H. Gong, S. Zeng, C. Yan et al., "Continuously tracing brain-wide long-distance axonal projections in mice at a one-micron voxel resolution," Neuroimage 74, 87-98 (2013).

31. T. Gensch, M. Wirth, "Determination of calcium concentrations in cells and tissue with fluorescence 
lifetime imaging (FLIM)," Proc. SPIE 7903, 790322 (2011).

32. J. Leppert, J. Krajewski et al., "Multiphoton excitation of autofluorescence for microscopy of glioma tissue," Neurosurgery 58, 759-767 (2006).

33. S. R. Kantelhardt, D. Kalasauskas, K. Konig et al., "In vivo multiphoton tomography and fluorescence lifetime imaging of human brain tumor tissue," J. Neuro-Oncol. 127, 473-482 (2016.)

34. L. Marcu, B. A. Hartl, "Fluorescence lifetime spectroscopy and imaging in neurosurgery," IEEE J. Sel. Top. Quantum Electron. 18, 1465-1477 (2012).

35. T. Timm, J. P. von Kries, X. Y. Li et al., "Microtubule affinity regulating kinase activity in living neurons was examined by a genetically encoded fluorescence resonance energy transfer/fluorescence lifetime imaging-based biosensor inhibitors with therapeutic potential," J. Biol. Chem. 286, 41711-41722 (2011).

36. J. L. Rinnenthal, C. Bornchen, H. Radbruch et al., "Parallelized TCSPC for dynamic intravital fluorescence lifetime imaging: Quantifying neuronal dysfunction in neuroinflammation," PLoS One 8, e60100 (2013).

37. C. Dysli, S. Wolf, K. Hatz et al., "Fluorescence lifetime imaging in stargardt disease: Potential marker for disease progression," Invest. Ophthalmol. Vis. Sci. 57, 832-841 (2016).

38. C. Dysli, S. Wolf, M. S. Zinkernagel, "Fluorescence lifetime imaging in retinal artery occlusion," Invest. Ophthalmol. Vis. Sci. 56, 3329-3336 (2015).

39. D. Schweitzer, L. Deutsch, M. Klemm et al., "Fluorescence lifetime imaging ophthalmoscopy in type 2 diabetic patients who have no signs of diabetic retinopathy," J. Biomed. Opt. 20, 061106 (2015).

40. U. Gehlsen, A. Oetke, M. Szaszak et al., "Two-photon fluorescence lifetime imaging monitors metabolic changes during wound healing of corneal epithelial cells in vitro," Graefes Arch. Clin. Exp. Ophthalmol. 250, 1293-1302 (2012).

41. J. Siegel, D. S. Elson, S. E. D. Webb et al., "Studying biological tissue with fluorescence lifetime imaging: Microscopy, endoscopy, and complex decay profiles," Appl. Opt. 42, 2995-3004 (2003).

42. Y. H. Sun, J. E. Phipps, J. Meier et al., "Endoscopic fluorescence lifetime imaging for in vivo intraoperative diagnosis of oral carcinoma," Microsc. Microanal. 19, 791-798 (2013).

43. H. Fatakdawala, S. Poti, F. F. Zhou et al., "Multimodal in vivo imaging of oral cancer using fluorescence lifetime, photoacoustic and ultrasound techniques," Biomed. Opt. Express 4, 1724-1741 (2013).
44. K. Konig, H. Schneckenburger, R. Hibst, "Timegated in vivo autofluorescence imaging of dental caries," Cell. Mol. Biol. 45, 233-241 (1999).

45. M. E. Zevalbs, S. K. Gayen, B. B. Das et al., "Picosecond electronic time-gated imaging of bones in tissues," IEEE J. Sel. Top. Quantum Electron. 5, 916-922 (1999).

46. P. Y. Lin, H. C. Lyu, C. Y. S. Hsu et al., "Imaging carious dental tissues with multiphoton fluorescence lifetime imaging microscopy," Biomed. Opt. Express 2, 149-158 (2011).

47. H. L. Wang, X. W. Liang, Y. H. Mohammed et al., "Real-time histology in liver disease using multiphoton microscopy with fluorescence lifetime imaging," Biomed. Opt. Express 6, 780-792 (2015).

48. J. Mizeret, T. Stepinac, M. Hansroul et al., "Instrumentation for real-time fluorescence lifetime imaging in endoscopy," Rev. Sci. Instrum. 70, 46894701 (1999).

49. D. Gorpas, H. Fatakdawala, J. Bec et al., "Fluorescence lifetime imaging and intravascular ultrasound: Co-registration study using ex vivo human coronaries," IEEE Trans. Med. Imag. 34, 156-166 (2015).

50. J. Bec, H. T. Xie, D. R. Yankelevich et al., "Design, construction, and validation of a rotary multifunctional intravascular diagnostic catheter combining multispectral fluorescence lifetime imaging and intravascular ultrasound," J. Biomed. Opt. 17, 106012 (2012).

51. H. Fatakdawala, D. Gorpas, J. W. Bishop et al., "Fluorescence lifetime imaging combined with conventional intravascular ultrasound for enhanced assessment of atherosclerotic plaques: An ex vivo study in human coronary arteries," J. Cardiovasc. Transl. Res. 8, 253-263 (2015).

52. J. E. Phipps, Y. H. Sun, M. C. Fishbein et al., "A fluorescence lifetime imaging classification method to investigate the collagen to lipid ratio in fibrous caps of atherosclerotic plaque," Lasers Surg. Med. 44, 564-571 (2012).

53. A. Ostman, "The tumor microenvironment controls drug sensitivity," Nat. Med. 18, 1332-1334 (2012).

54. L. V. Zholudeva, K. G. Ward, M. G. Nichols et al., "Gentamicin differentially alters cellular metabolism of cochlear hair cells as revealed by NAD $(\mathrm{P}) \mathrm{H}$ fluorescence lifetime imaging," J. Biomed. Opt. 20, 051032 (2015).

55. V. V. Ghukasyan, F. J. Kao, "Monitoring cellular metabolism with fluorescence lifetime of reduced nicotinamide adenine dinucleotide," J. Phys. Chem. C 113 11532-11540 (2009).

56. M. S. Roberts, Y. Dancik, T. W. Prow et al., "Noninvasive imaging of skin physiology and percutaneous 
penetration using fluorescence spectral and lifetime imaging with multiphoton and confocal microscopy," Eur. J. Pharm. Biopharm. 77, 469-488 (2011).

57. T. W. Prow, D. Liu, R. Faye et al., "Simultaneous imaging of metal nanoparticle penetration and metabolic changes in human skin using fluorescence lifetime imaging," Exp. Dermatol. 20, E873-E874 (2011).

58. S. Kawanabe, Y. Araki, T. Uchimura et al., "Applying fluorescence lifetime imaging microscopy to evaluate the efficacy of anticancer drugs," Methods Appl. Fluoresc. 3, 025006 (2015). 